\title{
Aspectos demográficos en comunidades indígenas de tres regiones de Colombia
}

\author{
Marion Piñeros-Petersen, M.C., M.Sc., ${ }^{(1)}$ Magda Ruiz-Salguero, Lic. en Estadística, M. en D emografía. ${ }^{(2)}$
}

\begin{abstract}
Piñeros-Petersen M, Ruiz-Salguero M. A spectos demográficos en comunidades indígenas de tres regiones de Colombia. Salud Publica Mex 1998;40:324-329.
\end{abstract}

\section{Resumen}

Objetivo. 0 btener indicadores demográficos para comunidades indígenas colombianas, localizadas en tres grandes regiones del país: la del Caribe, la amazónica y la andina. Material y métodos. Se analizaron las variables demográficas de una encuesta sobre conocimientos, actitudes y prácticas, realizada entre 1993 y 1994, con una muestra de 11522 indígenas. Resultados. El 45\% de la población es menor de 15 años; la tasa global de fecundidad es de 6.5 hijos por mujer, y la tasa de mortalidad infantil para 1990, de 63.3 por 1000 nacidos vivos. La esperanza de vida al nacer fue de 57.8 años para mujeres y de 55.4 años para nombres. Conclusiones. Los indicadores difieren sustancialmente del promedio nacional.Aunque pareciera que las poblaciones estudiadas están iniciando un proceso de transición demo gráfica, existen diferencias regionales marcadas, con niveles de fecundidad y mortalidad infantil en la región del Caribe muy superiores a las de las otras dos regiones.

Palabras clave: indios sudamericanos; demografía; fecundidad; mortalidad; Colombia

\author{
Piñeros-Petersen M, Ruiz-Salguero M. \\ Demographic features of indigenous \\ communities in three regions of Colombia. \\ Salud Publica Mex 1998;40:324-329.
}

\begin{abstract}
Objective. To obtain demographic indicators for some indigenous communities in Colombia situated in three different regions of the country: the $C$ aribbean, the Amazonic basin and the Andes. Material and methods. D emographic variables gathered in a KAP (knowledge, attitude and practices) survey among the indigenous population in 1993 and 1994 were analyzed. The survey included 11522 Indians. Results. $45 \%$ of the population is under age 15 ; overall rate of fertility is 6.5 children per wo man, and death rate in 1990 was 63.3 children per 1000 live births. Life expectancy at birth was 57.8 years for women and 55.4 years for men. Conclusions. The indicators differ substantially from the national ciphers. Although the indigenous population seems to be undergoing a process of demographic transition, there are marked differences between regions, with significantly higher fertility and infant mortality rates for the Caribbean region.
\end{abstract}

Key words: Indians, South American; demography; fertility; mortality; Colombia
C olombia vive un momento de profundos cambios en su organización política, y en el marco de la descentralización, los indicadores sociodemográficos se convierten en los elementos estratégicos para la planificación, la gestión y la evaluación de programas, así como para comparar los distintos grupos sociales. Cabe destacar entonces la importancia que reviste contar con información estadística oportuna, organizada y de buena calidad, especialmente para grupos minoritarios que requieren de la puesta en marcha de acciones y políticas específicas. Sin embargo, la obtención de estadísticas vitales en población indígena no es tarea

(1) Asesora de la Dirección Técnica de Censos del Departamento Administrativo N acional de Estadísticas para el proyecto Censo 2000, Santa Fé de Bogotá, Colombia.

(2) Asistente de Gerencia, Investigación y Evaluación de Profamilia, Santa Fé de Bogotá, Colombia.

Fecha de recibido: 25 de abril de 1997 - Fecha de aprobado: 22 de abril de 1998 electrónico: avilla@ multi.net.co 
fácil si se tiene en cuenta la ausencia de sistemas numéricos y la dificultad que plantea la interpretación de categorías de tiempo. Lo anterior privilegia el uso de métodos indirectos para estimar la mortalidad en este tipo de poblaciones. ${ }^{1}$

La población indígena de Colombia ha sido estimada con base en diversas fuentes, en cifras que van de los 450000 y 592000 individuos. ${ }^{2, *}$ El censo de 1993 revela que $2.2 \%$ de la población colombiana vive en áreas predominantemente indígenas, lo cual equivale a 823900 personas, teniendo en cuenta el censo ajustado por cobertura.*

Los censos de población indígena realizados en el país han presentado dificultades metodológicas, principalmente en la definición del ser indígena, por la multiplicidad de etnias existentes y por la gran dispersión que presentan los distintos grupos. ${ }^{3}$

Aun cuando se reconoce que el estado de salud de la mayoría de las comunidades indígenas colombianas es malo, es muy escasa la información que se tiene sobre estadísticas vitales e indicadores de salud. Algunos estudios puntuales ponen de manifiesto la existencia de profundas desigualdades, con tasas de mortalidad infantil en población indígena que superan ampliamente a las del resto de la población. ${ }^{4,5}$ Las diferencias en niveles de mortalidad entre población indígena y no indígena han sido documentadas también en otros países de América Latina, e incluso se afirma que la pertenencia a una población indígena supone una desventaja que persiste aun cuando mejoran las condiciones económicas y sociales. ${ }^{6}$

En el presente trabajo, resultado del análisis de la información demográfica obtenida en una encuesta sobre conocimientos, actitudes y prácticas en salud en comunidades indígenas, se corroboran las enormes diferencias que existen entre los indicadores demográficos de las poblaciones indígenas y los del resto del país; asimismo se observa una importante variación entre ellas mismas.

\section{Materiales y métodos}

Los datos provienen de la encuesta "Conocimientos, actitudes y prácticas en salud en comunidades indígenas" (CAPS), realizada en 1993-1994 por el Instituto Nacional de Salud en una muestra no probabilística

\footnotetext{
* Departamento Nacional de Planeación, División de Población. Población total e indígena, extensión territorial y distribución relativa de la población indígena respecto del total nacional. Santa Fé de Bogotá, enero de 1993. Documento no publicado.
}

de comunidades indígenas del país. Se seleccionaron los grupos étnicos más numerosos del país. La selección de asentamientos tuvo en cuenta factores como la accesibilidad, los costos, los problemas de orden público, así como el interés y la aceptación de la encuesta por parte de las autoridades indígenas.

En la encuesta se utilizaron dos formularios: uno para el hogar y la vivienda, y otro dirigido a mujeres en edad fértil. Se realizó la entrevista en 2158 hogares, de tal modo que se logró una cobertura global de $91 \%$. La población estudiada fue de 11522 personas. Para el análisis demográfico, la población se agrupó de la siguiente manera: en la región del Caribe se estudiaron 3040 indígenas de las etnias wayú y arhuaco, en 566 hogares; en la región andina, 4847 indígenas coyaima, paez y emberá (de montaña), en 896 hogares, y en la región amazónica, 3635 indígenas pertenecientes a 16 grupos étnicos (con predominio de los cubeo, desano, tukano y puinave), en 696 hogares.

Para la estimación de la mortalidad infantil (es decir, en el primer año de vida) se utilizó el método indirecto de Brass, que toma en cuenta la información de hijos nacidos vivos durante toda la vida de la mujer, y de hijos sobrevivientes al momento de la entrevista. Se basa en el supuesto de que la proporción de hijos muertos de las mujeres de una determinada edad es una aproximación de la mortalidad entre el nacimiento y una determinada edad promedio de los hijos de esas mujeres. Mediante un multiplicador $(\mathrm{k})$ que contempla el patrón de fecundidad de la población, se convierten las proporciones de hijos muertos en probabilidades de morir entre el nacimiento y una edad determinada. El método también permite estimar el año al cual corresponde ese nivel de mortalidad.

Para la mortalidad adulta se recurrió a la información sobre defunciones ocurridas en el hogar en un periodo de referencia; con esta última se construyeron las tasas centrales de mortalidad por sexo y grupos de edad. Con base en estas estimaciones de mortalidad infantil y adulta, se construyó la tabla de vida por sexo para el total de la población estudiada.

Para la fecundidad se utilizó el método de Brass basado en la declaración del número total de hijos nacidos vivos en el último año. Esta información permite calcular las tasas específicas de fecundidad por edad. Como la aplicación del método ha demostrado que las tasas calculadas con los nacimientos del último año generan una estructura por edad aceptable pero no así el nivel de fecundidad -particularmente en las poblaciones que no manejan el calendario-, se hace necesario aplicar un factor de ajuste derivado del cociente entre la paridez a una edad determinada (Px) y la fecundidad actual acumulada a esa edad (Fx). 
Para las distintas estimaciones se utilizó el Paquete para Estimaciones Demográficas PANDEM elaborado por CELADE.?

\section{Resultados}

\section{Características demográficas}

La estructura por edades para toda la población estudiada muestra una población predominantemente joven, pues $45 \%$ está compuesta por menores de 15 años (figura 1). Esta proporción, para el total de la población colombiana, es de 34\% según el censo de $1993 .{ }^{8}$

Por regiones, la población menor de quince años fue de $48 \%$ en la del Caribe, de $43 \%$ en la andina y de $45 \%$ en la amazónica.

Se observa una rectangularización de la pirámide en los grupos de edad de 0-15 que puede obedecer a un descenso de la fecundidad en los últimos 15 años, combinado con un descenso de la mortalidad infantil y juvenil. En las demás edades el angostamiento de la pirámide es rápido, lo que indica que estas cohortes han estado expuestas a mayores niveles de mortalidad y migración. En general, se observan menos mujeres que hombres salvo en algunas edades de atracción (por ejemplo, en el rango de 50-54 años), donde hay un abultamiento en el número de mujeres. El índice de masculinidad (número de hombres por cada 100 mujeres) es de 105 para la población de estos asentamientos y es mayor que 100 en casi todos los grupos de edad. Lo anterior puede obedecer a una mayor mortalidad o migración femenina, o bien, a una combinación de

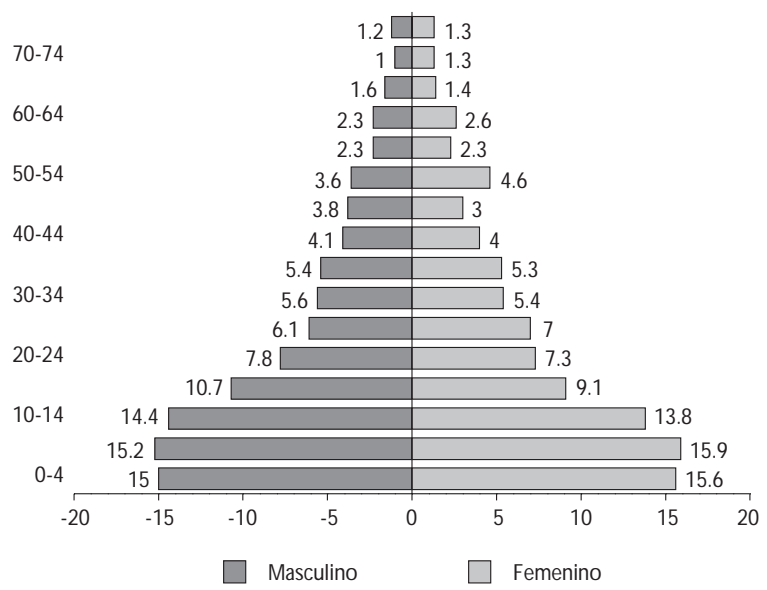

Figura 1. Estructura poblacional POR EDAD Y SEXo (\%). Encuesta CAPS. Comunidades indígenas DE Colombia, 1993-1994 ambos factores. El comportamiento de la distribución por sexo es similar al de la población rural del país afectada por la migración rural-urbana, predominantemente de adultos jóvenes, que se refleja en un índice de masculinidad de 113 hombres por cada 100 mujeres.

\section{Fecundidad}

La fecundidad estimada para los grupos analizados es bastante alta comparada con el contexto nacional. En Colombia las mujeres tienen en promedio tres hijos por mujer; ${ }^{9}$ las de estos asentamientos tienen más del doble de hijos (6.5 hijos por mujer), nivel que se observaba en el país 30 años atrás. Si se toma como referencia la fecundidad observada en el ámbito nacional dentro de las áreas rurales (4.3 hijos por mujer), se encuentra que en estas poblaciones indígenas las mujeres tienen en promedio dos hijos más, lo cual se ve reflejado en las altas tasas de fecundidad específicas en todas las edades (figura 2). No se investigó la fecundidad en el rango de 50-54 años pero, a juzgar por el nivel que presenta el grupo anterior, puede ser alta todavía.

$\mathrm{Al}$ interior de los grupos indígenas analizados se encuentran marcadas diferencias: mientras que las mujeres de la región andina tienen en promedio 5.5 hijos por mujer, las de la Amazonia tienen 6.9, y las de la región del Caribe, 8.3 hijos por mujer (figura 3). En el contexto nacional, la costa atlántica es también la región que presenta mayores tasas de fecundidad.

La tasa bruta de natalidad para el total de estos asentamientos es de 41 nacimientos por cada 1000 habitantes, la cual puede considerarse alta en comparación con el promedio nacional, que es de 26 por cada $1000 .{ }^{9}$ La relación niños-mujeres (RNM, menores de cinco años por cada 1000 mujeres de 15 a 49) es un indica-

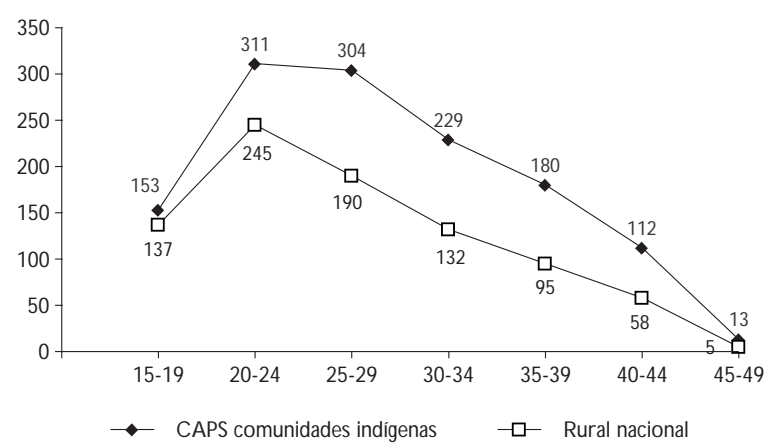

Figura 2.T ASAS DE FECUNDIDAD (POR 1000) POR GRUPOS DE EDAD. ENCUESTA CAPS. CoMUNidADES INDíGenAS Y rural nacional. Colombia, 1993-1994 


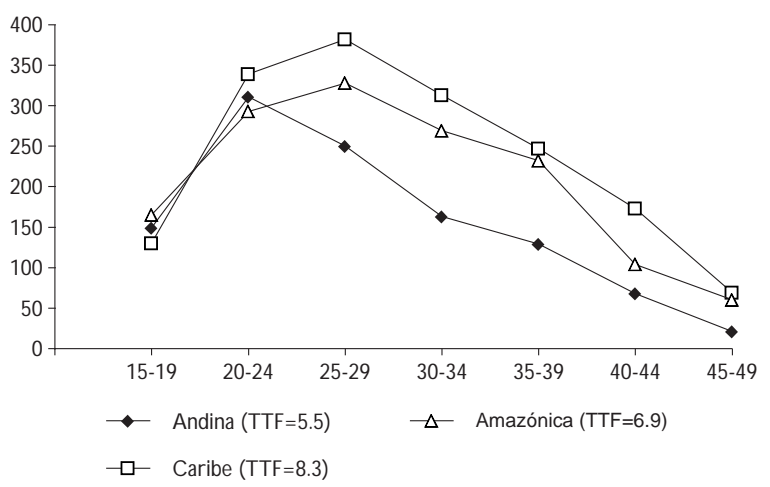

TTF: tasa total de fecundidad

Figura 3.TASAS ESPECÍfICAS DE FECUNDIDAD (POR 1000), por regiones. Encuesta CAPS. Comunidades indígenas de Colombia, 1993-1994

dor del nivel de fecundidad independiente de la declaración de nacimientos, puesto que se calcula con base en la estructura por edad de la población; para estas poblaciones se estimó en 762 niños por cada 1000 mujeres, lo que confirma el alto nivel de fecundidad.

\section{Mortalidad}

La mortalidad general, medida por la tasa bruta de mortalidad, fue de 10 defunciones por cada 1000 habitantes, la cual puede considerarse alta para una población con estructura por edad joven. La diferencia entre la tasa bruta de natalidad y la de mortalidad implica una tasa de crecimiento que en este caso se sitúa en 31 por cada 1000.

\section{Mortalidad infantil}

La mortalidad en el primer año de vida se ha considerado como uno de los principales indicadores de salud que además está estrechamente asociado con el nivel de desarrollo y las condiciones socioeconómicas de la población.

La tasa de mortalidad infantil (TMI) en Colombia, para el periodo 1989-1990, fue de 41.3 por cada 1000 nacidos vivos, y para la población rural fue de 50.5 por cada $1000 .{ }^{*}$ La TMI obtenida en la presente encuesta para 1984 fue de 87.7 por cada 1 000, y para 1990, de 63.3 por cada 1000 ; asimismo, se observaron grandes

\footnotetext{
* Departamento Nacional de Planeación, División de Población. Población total e indígena, extensión territorial y distribución relativa de la población indígena respecto del total nacional. Santa Fé de Bogotá, enero de 1993. Documento no publicado.
}

variaciones entre las distintas regiones (cuadro I). Los diferenciales por región son similares a los encontrados en la fecundidad, con mayores niveles para los asentamientos de la región del Caribe, donde el riesgo de morir en los niños es dos veces mayor que en los de las otras dos regiones.

La tendencia general para el total de la población estudiada es un descenso rápido de la TMI; sin embargo, para la región del Caribe la tasa presenta fluctuaciones pero se mantiene constante.

\section{Tabla de vida por sexo}

Con base en las preguntas indirectas en torno a la sobreviviencia de los hijos y al número de defunciones en el hogar se construyó la tabla de vida por sexo para el periodo 1992-1993, para el cual se estimó que la TMI es de 55 por cada 1000 . Uno de los resultados importantes es la mayor mortalidad femenina en los menores de un año y entre los de 1-4 años. Este diferencial es contrario a lo observado en la mayor parte de las poblaciones modernas. Considerando que es poco lo que se sabe acerca de las poblaciones indígenas como para contradecir este hallazgo, se conservó el diferencial en la construcción de la tabla.

La esperanza de vida para las mujeres resultó ligeramente mayor (57.8 años) que para los hombres (55.4 años). En la figura 4 se presentan las probabilidades de morir, por edad y sexo, de la población indígena de este estudio y de la población nacional para 1993. En la población indígena se observa que la mortalidad masculina adulta (a partir de los 10 años) es mayor que

\section{Cuadro I \\ Mortalidad infantil Según Regiones; TASAS POR 1 000. ENCUESTA CAPS. Comunidades indígenas de Colombia, 1993-1994}

\begin{tabular}{lllll} 
Año & \multicolumn{3}{l}{ Regiones } & \\
\cline { 2 - 5 } & Andina & Caribe & Amazónica & Total \\
\hline 1979 & & & & 116.7 \\
\hline 1980 & 77.7 & & 87.7 & \\
\hline 1982 & & 112.4 & 67.6 & 84.3 \\
\hline 1983 & 72.9 & & & \\
\hline 1984 & & 126.5 & 71 & 87.7 \\
\hline 1985 & 59.9 & & & 82 \\
\hline 1986 & & 107.6 & 80.3 & 74.5 \\
\hline 1988 & 57.6 & 105.5 & 54.6 & 63.3 \\
\hline 1990 & 40.7 & 111.7 & 48.6 & 55
\end{tabular}

*Proyectada con base en los resultados del presente trabajo 

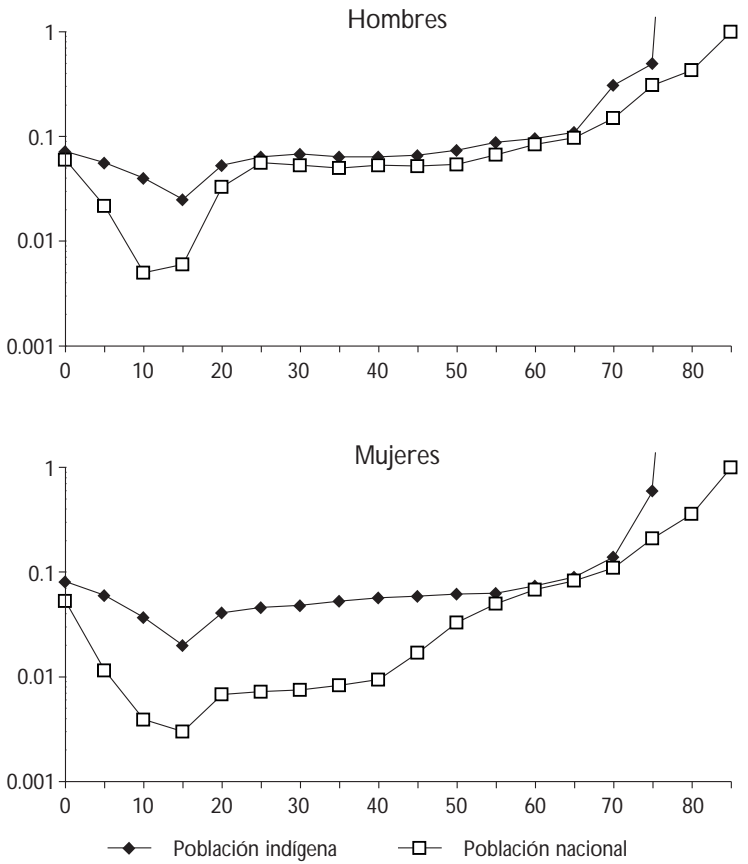

Figura 4. Probabilidades de morir, por sexo, en población indígena y nacional. Colombia, 1993

la femenina, aunque se juntan en las edades del periodo fértil, lo cual puede deberse a la mortalidad materna. De esta manera, la mayor mortalidad de las niñas es lo que explicaría el estrecho diferencial de la esperanza de vida al nacer. En el caso de Colombia se presenta el otro extremo, es decir, un diferencial de 8.9 años, con una expectativa de vida de 64.3 años para hombres y 73.2 para mujeres, explicado por la mayor mortalidad masculina que se presenta en todas las edades y especialmente en los adultos jóvenes a causa de la violencia.*

\section{Discusión}

El presente estudio mostró una población predominantemente joven, en concordancia con los hallazgos de otros estudios en poblaciones indígenas tanto colombianas como brasileñas y canadienses. ${ }^{4,5,10,11}$ Llama la atención el hecho de que haya más hombres que mujeres en la pirámide, lo cual podría deberse a una

\footnotetext{
* Departamento Administrativo Nacional de Estadística. Estimaciones demográficas y proyecciones nacionales de población 1950-2050. Santa Fé de Bogotá, febrero de 1998. Documento no publicado.
}

menor declaración de mujeres por factores culturales, así como a una mayor migración en algunos grupos donde las mujeres salen a las ciudades para emplearse en trabajos domésticos. Empero, el estudio no explica este fenómeno, y los datos poblacionales del censo indígena de 1993 seguramente aportarán elementos para su aclaración. Por otro lado, es probable que el abultamiento en el grupo de 50-54 años de las mujeres se deba a un sesgo en la declaración de edad por parte de los encuestadores, ante mujeres que no entienden claramente el español y que además manejan el concepto de tiempo de otra manera.

Las altas tasas de fecundidad que se encontraron -más altas que las observadas en otras poblaciones indígenas- podrían explicarse tomando en cuenta la gran importancia y el significado que tiene la función reproductiva entre las mujeres indígenas, la alta frecuencia de uniones libres sucesivas en algunas etnias ${ }^{12}$ y, posiblemente, la ausencia de uso de métodos anticonceptivos y la pérdida de mecanismos propios de control demográfico. Este patrón de alta fecundidad está asociado con intervalos intergenésicos cortos y una alta paridez que, combinados con edad avanzada de la madre dentro del periodo fértil, los convierte en nacimientos de alto riesgo para la madre y el niño. Estas altas tasas, sumadas al hecho de tener una menor accesibilidad a los servicios de salud y de que se observe una mayor frecuencia de atención del parto por personal no capacitado formalmente, hacen de la población indígena femenina en edad reproductiva un grupo especialmente vulnerable.

La mortalidad infantil, a pesar de encontrarse aún muy por encima del promedio nacional, ha disminuido significativamente. Sin embargo, se encontró una gran variación entre las distintas regiones. La TMI es un indicador bastante sensible de la accesibilidad, la utilización y la efectividad de los servicios de salud, particularmente de los perinatales, ${ }^{13} \mathrm{y}$ parece ser que el mayor impacto en la reducción de tasas de mortalidad infantil en población indígena no está dado tanto por mejoras en las condiciones socioeconómicas e higiénicas, sino principalmente por un mayor acceso y utilización de los servicios de salud y por haber adquirido algún tipo de conocimiento específico sobre medidas preventivas. ${ }^{6,14} \mathrm{Al}$ respecto cabe anotar que durante los últimos años se ha hecho un esfuerzo importante por aumentar la cobertura de los servicios de salud en las comunidades indígenas, particularmente en lo que se refiere a la capacitación de promotores de salud indígenas. La diferencia de mortalidad en relación con la accesibilidad a los servicios de salud estaría dada, a su vez, particularmente en enfermedades de etiología infecciosa. ${ }^{15}$ 
Asimismo, destaca la sobremortalidad femenina en los menores de cinco años; este fenómeno se observa también en algunas partes de Africa y de Asia, donde se ha correlacionado con factores como una menor actividad económica de las mujeres, el establecimiento de una vivienda exogámica al casarse y el pago de dote para la boda de las mujeres. ${ }^{16}$ En algunas comunidades indígenas colombianas se encuentran estos factores que podrían explicar la sobremortalidad femenina infantil; no obstante, es necesario realizar mayores estudios en ese sentido.

Este trabajo indica que en la población estudiada se está iniciando un proceso de transición demográfica donde, a pesar de las malas condiciones socioeconómicas, la TMI está disminuyendo, mientras que la tasa de fecundidad aún es alta, lo que determina un crecimiento importante de la población indígena. Este hecho plantea una reflexión ineludible, tanto para las comunidades indígenas como para los planificadores, en términos de la conservación de la cultura ante un crecimiento importante de la población.

\section{Referencias}

1. Gómez LC, Ruiz M. Las encuestas en la producción de información para el análisis de la mortalidad (la experiencia colombiana). Boletín de Estadística del DANE 1988;(425):190-219.

2. Arango R, Sánchez E. Los pueblos indígenas de Colombia (población y territorio). Santa Fé de Bogotá: Departamento N acional de Planeación, 1989.
3. Ruiz M, Bodnar Y. El carácter multiétnico de Colombia y sus implicaciones censales. En: Estudios sociodemográficos de pueblos indígenas. Santiago de Chile: CELADE/CID O B/FN UAP/ICI, 1994.

4. A rias $M$, Alcaraz $G, G$ álvez $A$. Situación de salud materno-infantil en asentamientos emberá catíos, Dabeiba-Antioquia, 1985-1986. Boletín Epidemiológico de Antioquia 1987;13(1):118-127.

5. Pachón X. Paez. En: Correa F, Pachón X, ed. Introducción a la ColombiaA merindia. Santa Fé de Bogotá: Instituto Colombiano de Antropología/ Editorial Presencia, 1987.

6. Robles A. Diferencias de salud materno-infantil entre las poblaciones indígenas y no indígenas de Bolivia y Guatemala. En: Estudios sociodemográficos de pueblos indígenas. Santiago de Chile: CELADE/CID O B/ FN UAP/ICI, 1994.

7. Centro Latinoamericano de D emografía/C omisión paraA mérica Latina y el Caribe. PAN D EM. Manual del usuario. Santiago de Chile: 0 rganización de las N aciones Unidas, 1988.

8. Departamento Administrativo Nacional de Estadística. Censo 1993. Resumen nacional. Santa Fé de Bogotá: DANE, 1996.

9.Profamilia. Encuesta $N$ acional de Demografía y Salud. Santa Fé de Bogotá: Profamilia, 1995.

10. Hern W. Health and demography of native amazonians: historical perspective and current status. Cadernos Saude Publica 1991;7(4):451-480. 11. Pelletier F. Encuesta sobre los Pueblos Autóctonos de Canadá, 1991: un reflejo de nuestra historia. En: Estudios Sociodemográficos de Pueblos Indígenas. Santiago de Chile: CELADE/CID O B/FN UAP/ICI, 1994.

12. Galvez A. Fecundidad y muerte: visión interdisciplinaria sobre los embera de Dabeiba. En: Memorias del V Congreso Nacional de Antropología. Santa Fé de Bogotá: UCFES, 1989:311-326.

13. W orld Health 0 rganization. D evelopment of indicators for monitoring progress towards health for all by the year 2000. Ginebra: W HO, Health for All Series, núm. 4, 1981.

14. Kundstadter P, Lennington S, Leepreecha P, Podhisita C, Laoyang M, Sae Thao $C$ et.al. $C$ auses and consequences of increase in child survival rates: ethnoepidemiology among the Hmong of Thailand. Hum Biol 1992;64(6):821-864.

15. Kunitz, S J. Public policy and mortality among indigenous populations of N orthern A merica and Australasia. Popul D ev Rev 1990;16(4):647-672. 16. Basu AM. Is discrimination in food really necessary for explaining sex differentials in childhood mortality? Popul Stud 1989;(2):193-211. 